\title{
Consumer acceptance of saturated fat-reduced dairy products: a novel approach for reducing intake of saturated fat at a population level
}

\author{
O. Markey ${ }^{1,2}$, A. Hargreaves ${ }^{2}$, C.C. Fagan $^{2}$, D. Vasilopoulou ${ }^{1,2}$, K.E. Kliem ${ }^{3}$, D.J. Humphries ${ }^{3}$, \\ K.G. Jackson ${ }^{1,2}$, D.I. Givens ${ }^{3}$, J.A. Lovegrove ${ }^{1,2}$ and L. Methven ${ }^{2}$ \\ ${ }^{1}$ Hugh Sinclair Unit of Human Nutrition and Institute for Cardiovascular and Metabolic Research, \\ ${ }^{2}$ Department of Food and Nutritional Sciences and ${ }^{3}$ Food Production and Quality Division, University of Reading, \\ Reading, RG6 6AP, UK
}

At a population level, it is important to reduce modifiable CVD risk factors, and a key strategy is a reduction in dietary saturated fatty acids (SFA). Despite recommendations to reduce intake of SFA to $<10 \%$ of total energy; this target is exceeded by the majority of adults and children ${ }^{(1)}$. Consequently, there is an urgent need to find effective novel dietary approaches for reducing SFA intake ${ }^{(2)}$. Replacing milk SFA with unsaturated fatty acids (FA), through plant oil supplementation of the bovine diet, offers a sustainable means of producing SFA-reduced dairy products ${ }^{(3)}$. This study evaluated consumer acceptance of SFA-reduced dairy products, when compared to commercially available alternatives.

Modified, SFA-reduced Cheddar cheese and butter were manufactured at pilot scale, using milk (54 g/100 g SFA and $32 \mathrm{~g} / 100 \mathrm{~g}$ cis-MUFA) that was produced from a $21 \mathrm{~d}$ high-oleic sunflower oil bovine supplementation period ${ }^{(4)}$. A series of four unbranded cheddar cheese and butter/spreads (SFA-reduced (modified); regular SFA (control), and two additional commercially available comparators varying in total fat content and FA composition) were presented to consumers in a single blinded, balanced order. Consumer liking and product replacement intent were assessed on hedonic category scales.

Our cohort (53 men, 69 women; age 39.8 (SD 13.7) years; BMI 25.3 (SD 4.1 ) kg/m²) were moderately health conscious, with $56 \%$ of consumers reporting that they often actively looked at health-related information on food labels when purchasing a new food. There was a significant difference in overall liking between the four cheese samples $(P<0 \cdot 0001)$, with the modified cheese being the least liked sample. A significant difference in overall liking between the four butter/spread samples was also evident $(P<0 \cdot 000)$, with the modified butter being the second most favoured variety. When asked about product replacement, $43 \%$ and $39 \%$ of consumers respectively, said they would buy the modified cheese and butter as an alternative to their habitual products. Hierarchal cluster analysis identified three clusters that were representative of different patterns of consumer liking of the cheese and butter samples. For cheese samples, the largest cluster (43\%) liked the modified and control cheese equally, but significantly less than the two commercial comparators (low-fat medium cheddar and low value own label mild cheddar). The second largest group (30\%) did not significantly discriminate between the cheese samples. There were no demographic distinction within the largest cluster; however the less-discriminating cluster $(30 \%)$ was predominantly from the higher socio-economic group (SEG). For butter, the largest cluster $(46 \%)$ liked the commercial spread (olive oil/butter blend) significantly more than the modified butter, but liking ratings for the modified butter were not significantly lower than for the low-fat commercial spreadable butter. The smallest group (18\%) were nondiscriminators who rated all products with high liking scores. The only demographic distinction fell within the smallest cluster (18\%) which had a higher proportion of younger consumers from a higher SEG.

The present study has illustrated that a high proportion of consumers prefer conventional dairy products to SFA-reduced versions when tasted in a blinded manner. This suggests that modified dairy products require further optimisation before they will be accepted as a vehicle for reducing SFA intake at a population level. Future work should examine how exposure to SFA-reduced dairy products, with and without accompanying health-related information, impacts on their acceptance.

This research was supported by the Medical Research Council, Arla Foods and AarhusKarlshamn (AAK) UK.

1. Department of Health (2011) National Diet and Nutrition Survey: Headline results from Years 1 and 2 (combined) of the rolling programme 2008/ 9-2009/10.

2. Markey et al. (2014) Nutr Bull 39 : 161-171.

3. Givens et al. (2009) Animal 3: 1067-74.

4. Markey et al. Proc Nutr Soc (In Press). 